\title{
Efeitos da prática da natação adap- tada sobre o perfil bioquímico e 0 estado de condicionamento físico de indivíduos com lesão medular
}

\section{The effects of adaptated swimming program on the biochemstry blood profile and physical fitness in individuals with spinal cord injury}

Patricia A. de Almeida1, Valéria R. C. Barbosa², Matheus C. Dias ${ }^{3}$, Cassiano M. Neiva ${ }^{4}$

\begin{abstract}
RESUMO
Introdução: A ausência de exercícios físicos gerada pela imobilização dos membros inferiores conduz a mudanças na composição corporal que geralmente estão associadas com o desequilíbrio da taxa metabólica que somados ao estado sedentário podem resultar em obesidade, diabetes mellitus e doenças cardiovasculares. Assim, a melhora do condicionamento físico pode contribuir para promoção de saúde e qualidade de vida desses indivíduos. Como existe um número muito reduzido de pesquisas nesse sentido, nossa proposta foi investigar os efeitos de um programa de natação adaptada, em protocolo intervalado, para pessoas com lesão medular, tendo como objetivo verificar a melhora do seu condicionamento físico e, conseqüentemente, de algumas variáveis bioquímicas importantes para a saúde. Metodologia: Participaram do estudo 17 indivíduos com lesão medular, sedentários, distribuídos em 2 grupos: 11 participantes do grupo treinamento (GT) e 6 do grupo controle (GC). No GT foi aplicado um protocolo de treinamento intervalado em natação, durante oito semanas consecutivas, 3 vezes por semana. O protocolo empregou a braçada do nado peito, nos períodos de trabalho de intensidade moderada a intensa, e a braçada do nado costas, nos períodos de recuperação ativa. O GC não participou de nenhuma atividade física. Em ambos os grupos foi realizada a coleta de sangue para a análise bioquímica, antes (avaliação) e após (reavaliação) o programa de natação. Resultados e Discussão: As concentrações de triglicerídeos, colesterol total e LDL-colesterol não apresentaram alterações significativas da avaliação para a reavaliação, em ambos os grupos. Entretanto no GT, na dosagem de HDL-colesterol foram observadas diferenças significativas $(p=0,0110)$, evidenciando uma melhora na fase pós-treinamento, o que não ocorreu no GC. Com relação ao estado de condicionamento físico, os resultados revelaram uma diferença significativa em relação ao tempo e à distância percorrida na água quando comparados com a fase pré-treinamento $(p<0,001)$, demonstrando uma grande
\end{abstract}

1. Mestre em Promoção de Saúde; Docente da Faculdade Calafiori São Sebastião do Paraíso - MG

2. Mestre em Psicologia - Fisioterapeuta da Clínica Escola de Fisioterapia da Universidade de Ribeirão Preto - UNAERP.

3. Especialista em Fisiologia do Exercício - Aluno Especial do Programa de Pós-Graduação da Faculdade de Medicina de Ribeirão Preto - Universidade de São Paulo - USP.

4. Livre Docente em Metabolismo, Fisiologia do Esforço, Nutrição Humana e Antropometria - Professor Adjunto MS5 e Pesquisador Chefe do Laboratório de Metabolismo e Fisiologia do Esforço - MEFE da Faculdade de Ciências da Universidade Estadual Paulista - UNESP / Orientador do Programa de Mestrado/Doutorado em Promoção de Saúde da Universidade de Franca.
Correspondência:

Rua Cerqueira César, 950 - ap1401

Ribeirão Preto - SP / CEP: 14010-130

E-mail: patriciaalves_al@yahoo.com.br

Artigo recebido em 28/01/2011 Aprovado para publicação em 1\%/12/2011 
melhora na capacidade de deslocamento com a braçada do nado peito e uma melhora significativa da função cardiorrespiratória. Conclusão: O programa de natação intervalado empregado, com intensidade de moderada à intensa, pode, mesmo em curto período de tempo, promover alterações positivas nos valores de HDL-colesterol dos indivíduos com lesão medular estudados, além de melhorar substancialmente seu condicionamento físico.

Palavras-chave: Medula Espinal /lesões. Natação. Treinamento intervalado. Condicionamento Físico Humano. HDL-Colesterol. Promoção da Saúde.

\section{Introdução}

Dentre as patologias com alta prevalência na população de deficientes físicos, destacam-se as doenças cardiovasculares e outras associadas ao estado de inatividade física ${ }^{1,2}$ que se configuram na Síndrome Metabólica. Sendo que, existem evidências que comprovaram uma maior incidência de fatores de riscos para doenças cardiovasculares em indivíduos jovens com lesão medular do que em pessoas da população em geral. . $^{3-8}$

A ausência de exercícios físicos gerada pela imobilização dos membros inferiores conduz a mudanças na composição corporal que geralmente são associadas com o desequilíbrio da taxa metabólica (redução da concentração plasmática de HDL, aumento do colesterol total, dos triglicérides e LDL; resistência à insulina) que somados ao estado sedentário podem resultar em obesidade, diabetes mellitus e doenças cardiovasculares. . $^{2,5,6,9,10}$

Conforme pressupostos da I Diretriz Brasileira de Diagnóstico e Tratamento da Síndrome Metabóli$\mathrm{ca}^{11}$, a atividade física regular ou o exercício físico diminuem o risco relacionado a cada componente da síndrome metabólica, à medida que o exercício físico reduz a pressão arterial, eleva o HDL-colesterol e melhora o controle glicêmico.

Duran et al. ${ }^{12}$ e Jacobs e Nash ${ }^{5}$ apontam que indivíduos com lesão medular que iniciam em programas de exercícios seguramente melhorarão não só as funções cardiovasculares e musculares, como também o perfil lipídico e glicêmico. No entanto, é extremamente reduzido o número de estudos experimentais que investigam o efeito do exercício físico nos parâmetros sanguíneos bioquímicos, indicativos de doenças relacionadas a alterações metabólicas comuns em indivíduos com lesão medular. ${ }^{5,12}$

No Brasil, destacam-se os estudos realizados por Silva et al. ${ }^{13}$ e Quintana e Neiva ${ }^{14}$. Silva et al. ${ }^{13}$ em seu estudo, com lesados medulares praticantes de exercícios físicos e lesados medulares sedentários, identificaram influências positivas do exercício para manter a normalidade de alguns parâmetros bioquímicos, como do triglicérides e da glicose plasmática, além da redução do peso corporal. Quintana e Neiva ${ }^{14}$, ao compararem sedentários com deficiência física e jogadores de basquete de cadeira de rodas, constataram nestes últimos menores níveis de deposição de gordura central, melhores resultados quanto ao controle glicêmico e alterações menos pronunciadas do perfil lipidêmico, indicando resultados positivos em relação aos fatores de risco para a síndrome metabólica.

Dos esportes praticados por pessoas com deficiência física, a natação é considerada por vários autores como um exercício que traz muitos benefícios, em especial, aos indivíduos com lesão medular, na medida em que favorece a vivência da liberdade de movimento. Além disso, ela pode ser usada como uma estratégia de redução do peso corporal, já que o consumo de energia durante a prática da natação prolongada é um dos mais elevados entre todas as atividades esportivas realizadas por indivíduos com deficiência física, o que constitui um fator importante para auxiliar na prevenção de problemas decorrentes do excesso de peso. ${ }^{15-21}$

Conforme sugere a literatura, a melhora do condicionamento físico contribui para promoção de saúde e qualidade de vida de indivíduos com lesão medular. ${ }^{2,3,5,8,12,13,20,22-26}$ Desta forma, nossa proposta foi investigar os efeitos de um programa de natação adaptada, em protocolo intervalado, para pessoas com lesão medular, tendo como objetivo verificar a melhora do seu condicionamento físico e, conseqüentemente, de algumas variáveis bioquímicas importantes para a saúde.

\section{Material e métodos}

O estudo, de caráter experimental, envolveu indivíduos com lesão medular da cidade de Ribeirão 
Preto-SP. Utilizou-se uma amostragem por conveniência devido à dificuldade de reunir uma população com tais características e, ainda, devido a dificuldade de adesão a um programa de pesquisa envolvendo treinamento físico, alvo deste estudo.

Participaram do estudo 17 indivíduos com lesão medular, com idade entre 22 e 63 anos, todos do sexo masculino. A idade cronológica e o nível de lesão não foram considerados como critério de inclusão devido às limitações em se obter um número satisfatório de indivíduos para amostra do estudo.

Os sujeitos do estudo foram distribuídos em 2 grupos: 11 participantes do grupo treinamento (GT) e 6 do grupo controle (GC). Essa divisão ocorreu espontaneamente pela desistência dos sujeitos em praticar natação, a qual foi motivada, segundo relatos dos participantes, por insegurança de estar no meio aquático, ou devido às complicações de controle da bexiga e intestino.

Para inclusão ao GT, foram adotados os seguin- tes critérios: ter movimentação completa ou parcial de alguns músculos dos membros superiores que os capacitem a realizar a braçada dos nados peito e costas; apresentar atestado médico liberando-os para prática de exercícios físicos; estarem reabilitados quanto à higiene pessoal; não estar praticando natação; estar pelo menos três meses sem praticar nenhum esporte. Para o GC, além da lesão medular, adotou-se apenas o sedentarismo como critério de inclusão.

Os participantes selecionados assinaram o Termo de Consentimento Livre e Esclarecido e concordaram voluntariamente em participar da pesquisa que seguiu rigorosamente as normas da Declaração de Helsinki e as precípuas da resolução CNS 196/96 do Ministério da Saúde. A pesquisa foi regularmente submetida a dupla aprovação em Comitês de Ética, regularmente registrados no CONEP: protocolo 136/07 e protocolo 202/07.

As descrições das características dos componentes de cada grupo seguem na Tabela 1.

\section{Tabela 1}

Casuística do Grupo de Treinamento e do Grupo Controle

\begin{tabular}{|c|c|c|c|c|c|}
\hline itos & Idade (anos) & $\begin{array}{l}\text { Nível de } \\
\text { Lesão }\end{array}$ & $\begin{array}{l}\text { Classificação } \\
\text { Neurológica }\end{array}$ & $\begin{array}{c}\text { Tempo de } \\
\text { Lesão }\end{array}$ & Etiologia \\
\hline
\end{tabular}

\section{Grupo de Treinamento}

\begin{tabular}{|c|c|c|c|c|c|}
\hline 1 & 31 & $\mathrm{~T} 10$ & ASIAA & 8 anos & Acidente Automobilístico \\
\hline 2 & 35 & $\mathrm{~T} 12$ & ASIAA & 9 anos & Projétil de Arma de Fogo \\
\hline 3 & 34 & T12-L1 & ASIAA & 2 anos & Acidente de Trabalho \\
\hline 4 & 35 & T4-T5 & ASIAA & 11 anos & Acidente Automobilístico \\
\hline 5 & 22 & $\mathrm{~T} 7-\mathrm{T} 8$ & ASIA C & 2 meses & Acidente Automobilístico \\
\hline 6 & 34 & $\mathrm{~T} 12$ & ASIAA & 8 anos & Trauma em Assalto \\
\hline 7 & 26 & C5 & ASIA B & 7 anos & Mergulho em Águas Rasas \\
\hline 8 & 26 & T12-L1 & ASIAA & 10 meses & Queda de Altura \\
\hline 9 & 36 & $\mathrm{~L} 1$ & ASIAA & 7 anos & Projétil de Arma de Fogo \\
\hline 10 & 47 & $\mathrm{~T} 12$ & ASIAA & 26 anos & Trauma em Acidente de Trabalho \\
\hline 11 & 63 & T3-T4 & ASIAA & 9 anos & Tumor \\
\hline \multicolumn{6}{|c|}{ Grupo controle } \\
\hline 12 & 26 & L1-L2 & ASIAA & 4 anos & Acidente Automobilístico \\
\hline 13 & 26 & $\mathrm{C} 4-\mathrm{C} 7$ & ASIA B & 4 anos & Acidente Automobilístico \\
\hline 14 & 32 & $\mathrm{~L} 1$ & ASIAA & 8 anos & Acidente Automobilístico \\
\hline 15 & 25 & T12-L2 & ASIAA & 7 anos & Projétil de Arma de Fogo \\
\hline 16 & 30 & L4 & ASIAA & 1 ano & Queda de Altura \\
\hline 17 & 45 & $\mathrm{~L} 1$ & ASIAA & 10 anos & Acidente Automobilístico \\
\hline
\end{tabular}

ASIA (American Spinal Injury Association) - A (lesão completa); B (lesão incompleta, preservação sensitiva não-motora abaixo do nível neurológico da lesão); C (lesão incompleta, preservação motora abaixo do nível neurológico, com a maioria dos músculos-chave abaixo desse nível com grau menor que 3) 
Primeiramente, os indivíduos foram submetidos à anamnese e os participantes do GT foram submetidos, também, à exame de eletrocardiograma no Centro Clínico da universidade aonde o estudo foi realizado. Depois, foi realizada a coleta de sangue de todos os sujeitos, para análise bioquímica, no Laboratório de Análise Clínicas da mesma universidade.

Na obtenção da amostra sanguínea foram seguidas as recomendações de 12 horas de jejum, sem consumo de bebida alcoólica nem de medicamentos pelo período de 24 horas. As amostras foram obtidas do sangue venoso por punção da veia cubital, no período da manhã. ${ }^{28}$ As análises bioquímicas da glicemia de jejum (GLI), dos triglicérides (TRI), do colesterol total (CT) e frações deste (HDL e LDL) foram realizadas em triplicata, considerando a média entre elas. ${ }^{29}$ O método de determinação da glicose adotado foi o enzimático colorimétrico da oxidase. Para o CT, TRI e HDL-colesterol foram aplicados o método colorimétrico enzimático. A fração de colesterol LDL-colesterol foi obtida pela fórmula de Friedewald. ${ }^{30}$

Após essa primeira coleta de amostras, os sujeitos passaram por uma semana de adaptação ao meio líquido com o objetivo de treinarem a transferência da cadeira de rodas para a borda da piscina e praticarem exercícios educativos da braçada do nado peito e costas, com o uso do colete de flutuação, para a familiarização do material utilizado no protocolo de treinamento. O estudo foi realizado no Campus da Universidade de Ribeirão Preto, em piscina descoberta medindo $17 \times 25 \mathrm{~m}$, aquecida a $27^{\circ} \mathrm{C}$.

Depois da fase de adaptação, para avaliar estado de condicionamento físico inicial dos participantes do estudo e qual seria a intensidade do exercício durante as sessões, foi aplicado um teste de esforço com a utilização da Escala Subjetiva de Esforço de Borg. De acordo com alguns pesquisadores, existem dados que indicam que a percepção subjetiva de esforço pode ser usada como um indicador da intensidade do esforço em exercícios aquáticos. ${ }^{31,32,33}$

O teste de esforço foi aplicado individualmente e consistiu em realizar a braçada do nado peito, com a utilização do colete de flutuação, o mais rápido possível até a exaustão. Cada participante fazia um prévio alongamento e recebia explicações sobre a utilização da Escala de Percepção Subjetiva de Esforço. Iniciava o teste com a autorização verbal do professor que cronometrava o tempo de execução do teste. No instante de interrupção do nado, era registrado o tempo de exercício, a distância percorrida, a percepção sub- jetiva de esforço de acordo com a Escala de Borg e a frequência cardíaca (FC). A FC foi monitorada com frequencímetro modelo Polar F6 (POLAR Electro Oi - Filand).

O programa de natação foi elaborado seguindo alguns critérios de exercícios recomendados para indivíduos com lesão medular, elaborados por Jacobs e Nash5: são necessárias de 3 a 5 sessões semanais de exercícios, com duração de 20 a 60 minutos, numa intensidade de 50 a $80 \%$ do VO2pico, utilizando-se tipos de exercícios como natação, ergômetro de braço, esportes de cadeira de rodas, circuito de treinamento de resistência, etc.

Ainda segundo Jacobs e Nash ${ }^{5}$, essas prerrogativas sugerem que a intensidade alvo do exercício deve produzir respostas da FC equivalentes a 50-80\% da FCpico do indivíduo, sendo que, a frequência e a duração dos exercícios devem ser aumentadas gradativamente.

Uma semana após o teste de esforço, foi aplicado um protocolo de treinamento intervalado em natação, durante oito semanas consecutivas, com duração de 40 minutos cada sessão, três vezes por semana. Cada sessão foi composta de 5 minutos de alongamento fora da piscina, de 20 a 25 minutos de treinamento e o restante de atividades livres, como natação sem coletes ou atividades recreativas, até completar os 40 minutos.

Nas três primeiras semanas, os praticantes realizavam as sessões de treinamento na largura da piscina, ou seja, percorriam a distância de $17 \mathrm{~m}$ (medida da piscina $17 \times 25 \mathrm{~m}$ ). A partir da quarta semana de treinamento, as sessões foram realizadas no comprimento da piscina, $25 \mathrm{~m}$. Esse modelo de protocolo foi adaptado ao condicionamento físico do grupo de uma forma geral, e o aumento gradativo de esforço físico foi elaborado conforme o condicionamento físico da maioria do grupo.

O protocolo de treinamento intervalado empregou a braçada do nado peito nos períodos de trabalho de intensidade moderada a intensa, que corresponderia de 12 a 15 da Escala de Borg, e a braçada do nado costas nos períodos de recuperação ativa, com uma pequena pausa de recuperação passiva na borda da piscina, até completar o tempo de período de recuperação proposto para cada semana de treinamento.

As sessões foram constituídas por vários períodos de trabalho e de recuperação durante o tempo estipulado previamente que variou de 20 a 25 minutos: as $1^{\mathrm{a}}$ e $2^{\mathrm{a}}$ semanas as sessões de treinamento tiveram 
duração de 20 minutos, sendo que, o período de trabalho foi 30 segundos e o período de recuperação foi 1 minuto e 30 segundos, evoluindo para 1 minuto no período de trabalho e 30 segundos no período de recuperação, na $8^{a}$ semana, na qual as sessões tiveram 25 minutos de duração.

Os participantes realizaram as sessões de treinamento coletivamente. Ao som do apito, todos iniciavam a braçada do peito, cada um em seu ritmo, tentando atingir o número 15 da Escala de Borg. No segundo apito, todos mudavam de decúbito e realizaram a braçada do nado costas até atingir a borda.

\section{Análise estatística}

Inicialmente, os resultados do GT e do GC foram submetidos a um tratamento descritivo, em que foram calculados os valores da média aritmética, desvio padrão, faixa de extensão (valor máximo e mínimo) e coeficiente de variação (CV). Em ambos os grupos, para comparar os dados da análise bioquímica obtidos na fase pré (avaliação) com os dados da fase pós treinamento (reavaliação) foi utilizado o Teste $t$ de Student pareado. Para comparar os dados do GT com dos dados do GC foi utilizada o Teste t de Student para dados independentes. ${ }^{34}$

Com relação à avaliação do estado de condicionamento físico dos sujeitos do GT, utilizou-se o teste de Wilcoxom, específico para dados não paramétricos, para análise e comparação dos valores obtidos no teste de esforço para a distância, o tempo e a Escala de Borg. Já para os valores da FC do teste, utilizou-se o teste $t$ Student para dados pareados e paramétricos 34.

Para todas as análises, adotou-se um nível de significância de $\alpha=0,05$. A estatística descritiva e os testes estatísticos foram realizados através do programa Graph Pad Prism 5.0.

\section{Resultados}

Os resultados da análise bioquímica observados em ambos os grupos, na avaliação (fase inicial) e na reavaliação (fase pós-condicionamento do GT), estão apresentadas na Tabela 2.

De acordo com os dados observados na Tabela 2, verifica-se que a GLI (glicemia de jejum), os TRI (triglicérides), o CT (colesterol total) e LDL-colesterol (lipoproteína de baixa densidade) não apresenta-

Tabela 2

Concentração plasmática das variáveis bioquímicas estudadas no GC e no GT

\begin{tabular}{|c|c|c|c|c|c|c|c|}
\hline \multirow[b]{2}{*}{$\begin{array}{l}\text { Parâmetros } \\
(\mathrm{mg} / \mathrm{dl})\end{array}$} & \multicolumn{3}{|c|}{ Avaliação } & \multicolumn{3}{|c|}{ Reavaliação } & \multirow[b]{2}{*}{$\mathrm{P}$} \\
\hline & $\begin{array}{c}\text { Média e } \\
\text { Desvio Padrão }\end{array}$ & $\begin{array}{l}\text { Faixa de } \\
\text { Extensão }\end{array}$ & $\begin{array}{l}\mathrm{CV} \\
(\%)\end{array}$ & $\begin{array}{c}\text { Média e } \\
\text { Desvio Padrão }\end{array}$ & $\begin{array}{l}\text { Faixa de } \\
\text { Extensão }\end{array}$ & $\begin{array}{l}\mathrm{CV} \\
(\%)\end{array}$ & \\
\hline \multicolumn{8}{|c|}{ Grupo Controle } \\
\hline GU & $100 \pm 16,77$ & $84-125$ & 16,77 & $91,33 \pm 9,05$ & $79-102$ & 9,91 & 0,069 \\
\hline TRI & $93,67 \pm 50,37$ & $36-181$ & 46,27 & $97,83 \pm 17,20$ & $74-127$ & 17,58 & 0,415 \\
\hline $\mathrm{CT}$ & $176,3 \pm 52,36$ & $108-256$ & 29,69 & $168,5 \pm 26,17$ & $124-206$ & 15,53 & 0,323 \\
\hline HDL & $32,83 \pm 4,40$ & $27-38$ & 13,40 & $34,33 \pm 3,50$ & $29-38$ & 10,20 & 0,268 \\
\hline LDL & $123 \pm 43,11$ & 71 - 182 & 35,06 & $114,6 \pm 24,11$ & $80,2-155$ & 21,03 & 0,269 \\
\hline
\end{tabular}

Grupo Treinamento

\begin{tabular}{cccccccc} 
GLI & $90,36 \pm 7,69$ & $81-107$ & 8,50 & $94,36 \pm 15,8$ & $61-111$ & 16,74 & 0,162 \\
TRI & $124,5 \pm 57,58$ & $43-240$ & 46,27 & $113,6 \pm 77,98$ & $17-308$ & 68,63 & 0,232 \\
CT & $164,6 \pm 34,47$ & $120-226$ & 20,94 & $176,4 \pm 48,04$ & $123-289$ & 27,24 & 0,066 \\
HDL & $34,09 \pm 5,87$ & $25-43$ & 17,23 & $43,45 \pm 11,74$ & $28-64$ & 27,02 & $0,011^{*}$ \\
LDL & $105,7 \pm 29,51$ & $63-151$ & 27,92 & $110,2 \pm 39,99$ & $68-194$ & 36,28 & 0,252 \\
\hline
\end{tabular}

$* \mathrm{p}<0,05$ estatisticamente significativo 
ram alterações significativas nos momentos de avaliação e reavaliação para ambos os grupos. No GT, na dosagem de fração de colesterol HDL (lipoproteína de alta densidade) foram observadas diferenças significativas $(\mathrm{p}=0,011)$.

Na comparação entre os grupos GC e CT, a única variável que sofreu alteração significativa foi a dosagem de fração de colesterol HDL ( $\mathrm{p}=0,043)$ da fase inicial (avaliação), em que seus valores para as variáveis não apresentavam diferença significativa, para a fase pós-programa de condicionamento físico (reavaliação).

Com relação ao condicionamento físico do GT, após oito semanas de treinamento, os resultados médios obtidos no teste de esforço demonstraram uma melhora significativa $(\mathrm{p}<0,001)$ em relação à distância (metros) percorrida na água (Gráfico 1) e ao tempo (segundos) de duração do nado peito (Gráfico 2), quando comparados com a fase pré-treinamento. Já os resultados médios da Escala de Borg (Gráfico 3), antes e após o treinamento intervalado em natação, não sofreram nenhuma alteração significativa.

Com relação à FC aferida no momento final do teste de esforço, os dados dos sujeitos estão apresentados na Tabela 3 a seguir, juntamente com os resultados da percepção subjetiva de esforço obtidos através da Escala de Borg.

Tanto a média da \% da FC obtida ao final do Teste de Esforço, quanto a média dos dados obtidos na Escala de Borg não sofreram alterações significativas da avaliação para a reavaliação. Não houve também correlação da \% de FC ao final do teste e a percepção subjetiva de esforço obtida pela Escala de Borg, tanto na avaliação $(\mathrm{r}=0,04)$ quanto na reavaliação $(r=0,09)$ do Teste de Esforço.

\section{Discussão}

Conforme os dados apresentados na Tabela 1, a GLI, os TRI, o CT e a LDLcolesterol não apresentaram alterações significativas tanto no GC como no GT.

\section{Gráfico 1 - Comparação da distância média $(\mathrm{m})$ percorrida na avaliação do condicionamento físico do GT}

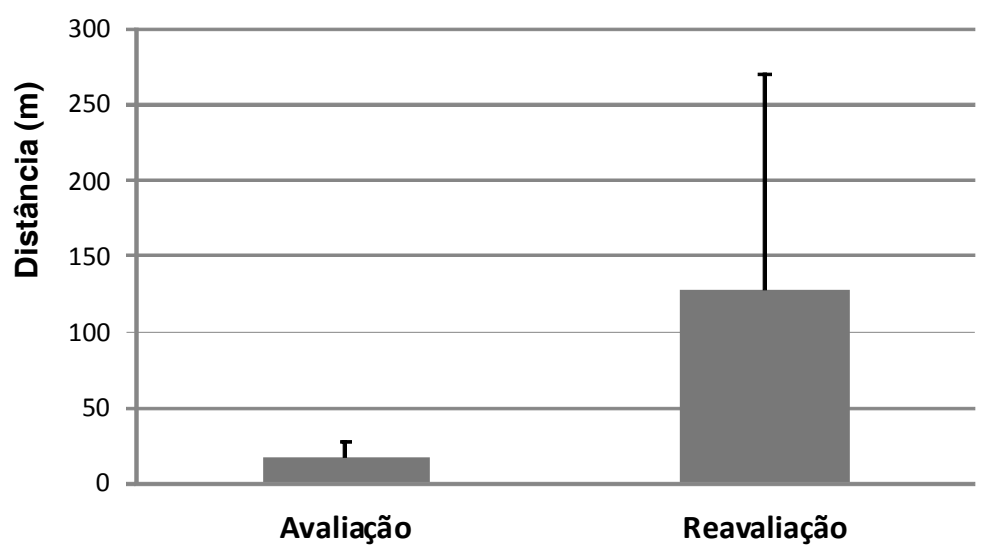

Gráfico 2 - Comparação do tempo médio (s) de duração na avaliação do condicionamento físico do GT

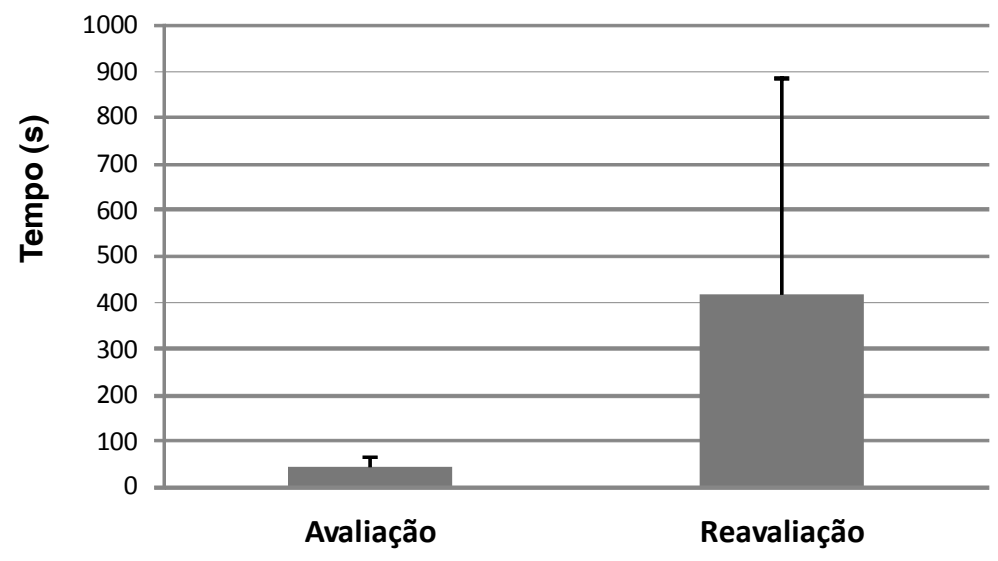

Gráfico 3 - Gráfico 3 - Comparação dos resultados médios
obtidos na Escala de Borg na avaliação do
con dicionamento físico do GT

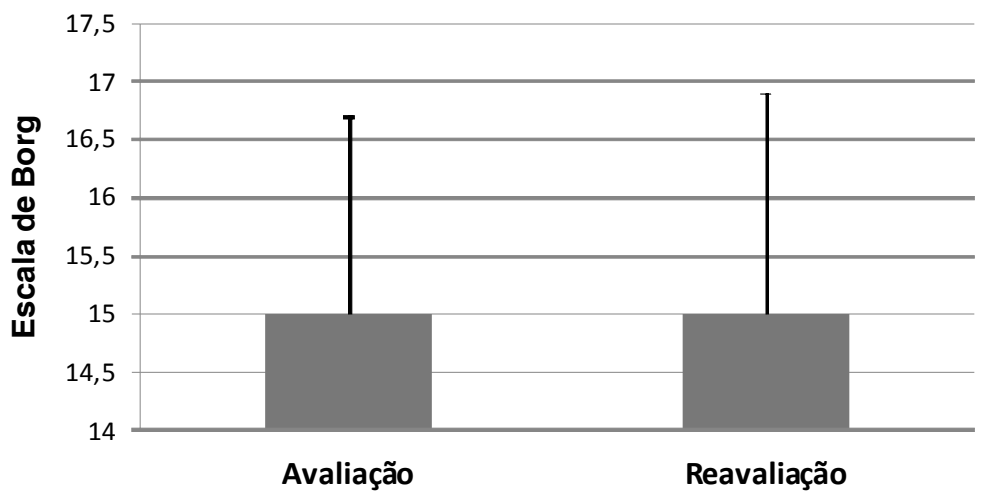


Tabela 3

Média, desvio padrão e coeficiente de variação da FC e da Escala de Borg obtidos na avaliação e reavaliação do Grupo Treinamento

\begin{tabular}{lcccccc}
\hline & \multicolumn{2}{c}{ Avaliação } & & \multicolumn{2}{c}{ Reavaliação } & P \\
\cline { 2 - 3 } & Média \pm DP & CV(\%) & & Média \pm DP & CV(\%) & 0,3395 \\
FC final do teste & $152 \pm 31$ & 20 & & $149 \pm 27$ & 18 & 0,3186 \\
\% da FC máx & $82 \pm 16$ & 19 & & $80 \pm 13$ & 16 & 0,3070 \\
Escala de Borg & $15 \pm 2$ & 11 & & $15 \pm 2$ & 13 & \\
\hline
\end{tabular}

Já no GT, na dosagem de fração de colesterol HDL foram observadas diferenças significativas $(\mathrm{p}=0,0110)$, evidenciando uma melhora na fase pós-condicionamento, o que não ocorreu no GC.

Os resultados que não demonstraram diferenças significativas, entre as fases de avaliação e reavaliação do GT, podem ser parcialmente explicados pela falta de controle de hábitos alimentares ${ }^{35}$ e talvez pela curta duração do protocolo de treinamento. Isso porque, segundo alguns pesquisadores, programas com mais de 12 semanas de duração são capazes de promover a redução do LDL-colesterol e do TRI, e aumento do HDL-colesterol ${ }^{36,37,38}$, o que foi confirmado por Lavis et al. ${ }^{2}$ na população de lesão medular.

No entanto, de acordo com Kraus et al. ${ }^{39}$ e Yuan et al. ${ }^{38}$ são justamente os níveis mais altos de exercício que promovem efeitos positivos no perfil lipídico. Fato este observado em estudo realizado por Durán et al. ${ }^{12}$, em que pacientes com lesão medular submetidos a um programa de resistência aeróbia, durante 16 semanas, com intensidade do exercício correspondente ao 10 da Escala de Borg (baixa intensidade), não apresentaram melhora estatisticamente significativa em nenhuma das variáveis bioquímicas estudadas (CT, TRI, HDL e LDL).

Em estudo realizado por Silva et al. ${ }^{13}$, com o objetivo de comparar os mesmos parâmetros bioquímicos que o nosso estudo, em indivíduos com lesão medular, os pesquisadores encontraram resultados semelhantes aos nossos. Os sujeitos praticantes de exercícios físicos (natação ou basquete) foram comparados com sedentários. Os resultados referentes à concentração plasmática de LDL-colesterol e TRI não apresentaram diferenças estatisticamente significativas entre os grupos. Em contrapartida, o HDL-colesterol apresentou valores abaixo do limite de normalidade em ambos os grupos.

Alguns pesquisadores constataram que o exercício pode exercer um efeito positivo no aumento do
HDL, porém não está completamente dimensionada a quantidade e a intensidade do exercício necessária para que isso ocorra. ${ }^{13,25,40}$

Rennie et al. ${ }^{41}$, Schwingel et al. ${ }^{42}$ e Pádua et al. ${ }^{43}$ constataram que as atividades mais vigorosas é que estão significativamente associadas com o aumento da concentração de HDL-colesterol e melhora de outros fatores relacionados à síndrome metabólica. Os nossos resultados também sugerem que, mesmo em pouco período de prática de exercícios, se a intensidade for de moderada a intensa, essa pode elevar os valores da concentração sanguínea de HDL-colesterol, o que talvez possa não ter ocorrido nos estudos de Silva et al. ${ }^{13}$ e Duran et al. ${ }^{12}$ devido à baixa intensidade dos exercícios.

O protocolo de exercícios empregado em nosso estudo, de intensidade moderada a intensa, foi capaz de promover alterações significativas na concentração de HDL-colesterol, sem contudo, alterar as demais variáveis, o que poderia ocorrer caso o protocolo fosse mais duradouro ou se a intensidade fosse mais ainda maior.

Os sujeitos do GT tiveram uma melhora significativa no condicionamento físico, da fase inicial para a fase pós-condicionamento, o que é demonstrado nos Gráficos 1, 2 e 3. Esse aumento, contudo, não refletiu em significativas e benéficas alterações bioquímicas para a maior parte das variáveis estudadas. Em relação à glicemia, a ausência de uma alteração significativa (em especial, uma diminuição de seus valores) pode estar relacionado com o estado inflamatório ${ }^{44,45}$ comum em indivíduos com lesão medular e caracterizado em boa parte nos sujeitos de nosso estudo. Essa condição que, eleva a glicemia, pode contrastar com os efeitos normo e hipo-glicemiantes do treinamento físico.

Outro aspecto a se considerar, é o fato de que os valores glicêmicos dos sujeitos já na fase pré encontravam dentro das razões normais de variação, o 
que minimiza os efeitos hipoglicemiantes terapêutico do treinamento.

Em relação às demais variáveis bioquímicas analisadas, Jacobs e $\mathrm{Nash}^{5}$, em estudo de revisão de literatura a respeito dos exercícios recomendados para lesados medulares, não verificaram alterações no CT dessa população após um programa de exercícios. Além disso, os autores constataram que os níveis de LDL-colesterol seguem os mesmos padrões típicos da população geral e não são necessariamente influenciados pelo nível de lesão.

Segundo os autores revisados por Jacobs e Nash5, o achado mais consistente se refere ao consenso de que o HDL-colesterol é baixo na população sedentária com lesão medular, cujos níveis estão inversamente associados com os riscos cardíacos. De acordo com Silva et al. ${ }^{13}$, alguns pesquisadores acreditam que a interrupção de comunicação no sistema nervoso autônomo altera a concentração de HDLcolesterol e, mesmo com um programa de exercícios, essa concentração tende a permanecer diminuída.

Em nossos achados, podemos novamente considerar que a intensidade, de moderada a intensa empregada no protocolo, foi eficiente em promover as alterações favoráveis nas concentrações favoráveis de HDL-colesterol contrariando o consenso apresentado por Silva et al. ${ }^{13}$ Contudo, provavelmente a falta de um acompanhamento alimentar adequado (o que não foi adotado em nosso estudo) pode ter contribuído para a ausência de alterações das demais variáveis lipídicas, visto que o consumo excessivo de alimentos com alto teor de glicose e lipídios pode interferir diretamente nesse quadro ${ }^{46}$, mesmo em indivíduos treinados.

Na comparação entre os grupos, a dosagem de fração de colesterol HDL apresentou diferenças significativas ( $\mathrm{p}=0,043)$ da fase inicial (avaliação), em que seus valores para as variáveis não apresentavam diferença significativa, para a fase pós-programa de condicionamento físico (reavaliação), evidenciando uma melhor condição do GT em relação ao GC na fase da reavaliação. Este mesmo resultado pode também ser observado na reavaliação do GT que foi demonstrado na Tabela 2.

$\mathrm{O}$ aumento de risco de ataque cardíaco em sedentários com lesão medular é de $60 \%$ a $90 \%$ maior do que em indivíduos aptos fisicamente, do mesmo sexo, idade, peso e altura. ${ }^{9}$ É bem conhecida a correlação entre baixos níveis de HDL-colesterol e aumento do risco de desenvolver doenças cardíacas. O HDLcolesterol menor que $40 \mathrm{mg} / \mathrm{dl}$ é por si só um fator de risco para doenças cardíacas, e o inverso, altos níveis de HDL-colesterol tem mostrado um efeito protetor. ${ }^{2}$

As alterações nos lipídeos frequentemente encontradas em indivíduos sedentários com lesão medular, como já foram mencionadas anteriormente, em estudos realizados por Jassen et al. ${ }^{3,23}$, estão associadas às causas comuns que também provocam essas alterações em pessoas da população em geral, como a falta de atividade física, o excesso de peso e de gordura corporal.

Em suas pesquisas, os autores verificaram que nenhuma das variáveis lipídicas estudadas em indivíduos com lesão medular estava relacionada com o nível de lesão. No entanto, o que acontece com esses indivíduos é que o excesso de peso, decorrente do sedentarismo, e o envelhecimento propiciam condições favoráveis para o surgimento precoce de valores bioquímicos alterados. Para tanto, os indicadores mais importantes para o risco de doenças coronárias são idade, tabagismo, etilismo, tecido adiposo e sedentarismo.

Em estudo com indivíduos com lesão medular, Janssen et al. ${ }^{23}$ afirmam que embora a capacidade física seja determinada por alguns fatores que não podem ser alterados, como idade, sexo e nível de lesão, ela pode melhorar substancialmente com a prática regular de exercícios físicos, o que pudemos observar em nosso estudo com relação à regularidade $\mathrm{e}$ intensidade do exercício.

Nesse sentido, observamos que os valores obtidos no teste de esforço para estimar o estado de condicionamento físico dos sujeitos do GT, na avaliação e na reavaliação, apresentados nos Gráficos 1, 2 e 3, demonstraram uma grande melhora nesse aspecto, quando o tempo de execução do exercício e a distância percorrida são avaliados e comparados com a Escala de Borg.

Após oito semanas de treinamento, os resultados demonstraram uma diferença significativa em relação à distância percorrida na água, quando comparados com a fase pré-treinamento $(\mathrm{p}<0,001)$. $\mathrm{O}$ aumento margeou a casa dos $700 \%$ o que se apresenta como um resultado extremante relevante na caracterização de uma melhora no estado de condicionamento físico.

O aumento da distância percorrida pode, em parte, ser explicado por uma melhora da eficiência mecânica dos membros superiores do nado peito, adquirida pelos sujeitos durante o protocolo. É importante lembrar ainda que, a grande magnitude da elevação 
média da distância percorrida é um pouco comprometida em função do desvio forçado por 3 sujeitos que tiveram resultados excepcionais. Entretanto, quando os resultados desses sujeitos são extraídos do cômputo geral, a variação ainda é maior do que $300 \%$, o que revela uma sensível melhora dessa variável demonstrando a eficiência do protocolo de treinamento em elevar a capacidade de deslocamento com a braçada do nado peito.

Em relação ao tempo de duração do exercício após o treinamento, o Gráfico 2 mostra uma elevação em torno de $890 \%$ em relação ao observado na avaliação inicial. Mesmo quando excluídos os resultados dos 3 sujeitos, já citados, que elevaram o desvio padrão, o aumento da capacidade de execução do exercício margeou a casa dos $350 \%$, resultados estes que demonstram uma melhora significativa $(\mathrm{p}<0,001)$ da função cardiorrespiratória, visto que a condição física inicial dos sujeitos não permitiu que conseguissem manter a braçada do peito por mais de 1 minuto e 19 segundos.

Contudo, a grande magnitude do aumento de distância $(700 \%)$ e de tempo (890\%) pode ser explicada, provavelmente, por uma grande e determinante melhora no estado de condicionamento físico. Dois fatores nos apontam para isso: a melhora da eficiência mecânica dos nados peito e costas dos indivíduos com lesão medular e o aumento do tempo de perdurance na atividade, que representa maior resistência muscular ao esforço, resultado clássico da melhora do condicionamento.

Com relação à Escala de Borg (Gráfico 3), os resultados demonstraram que a percepção subjetiva de esforço não se apresentou como uma ferramenta sensível para a avaliação da melhora do condicionamento físico em indivíduos com lesão medular ( $\mathrm{p}=0,743)$, especialmente, quando correlacionada com as demais variáveis aqui observadas.

Contudo, é possível considerar também que a manutenção do mesmo nível na escala, entre as duas fases testadas, ajuda-nos a reforçar a ideia do ganho de condicionamento físico por esses sujeitos em função do treinamento empreendido. Isso porque os sujeitos na reavaliação conseguiram manter o exercício por mais tempo, percorreram uma distância maior, e a média da sensação de esforço do grupo não se alterou da avaliação para a reavaliação, como está demonstrado no Gráfico 3.

A média da \% da FC obtida ao final do Teste de Esforço (mensurada com o propósito de possível correlação com a Escala de Borg) não sofreu alterações significativas $(\mathrm{p}=0,76)$ da avaliação para a reavaliação, o que demonstra que a FC não é um bom indicador da melhora do condicionamento físico, visto que o tempo médio e a distância média percorrida durante o teste aumentaram significativamente após as oito semanas de treinamento. Além disso, no teste de esforço, não houve também correlação da \% de FC ao final do teste e a percepção subjetiva de esforço obtida pela Escala de Borg.

Embora alguns autores apontem que a FC e a percepção subjetiva de esforço possam ser utilizadas como indicadores de intensidade de esforço no meio líquido ${ }^{32}$, em nosso estudo isso não ocorreu. $\mathrm{O}$ fato dos sujeitos não terem mobilidade de membros inferiores e terem realizado exclusivamente a braçada com o uso do colete de flutuação pode ter interferido nessas variáveis. Segundo Bodner e Rhodes ${ }^{47}$, o movimento contínuo dos membros superiores e dos ombros promove grande estimulação do plexo nervoso braquial, o que parece estimular o aumento da FC de forma demasiada, sem que isso implique necessariamente em uma relação direta com a intensidade metabólica do esforço.

Além disso, segundo Graef e Kruel ${ }^{32}$, no meio aquático, tanto durante o repouso como durante o exercício, a FC sofre influência de fatores como a posição do corpo, a profundidade de imersão, a temperatura da água, a FC de repouso e a diminuição do peso hidrostático.

Os resultados do tempo e da distância percorrida durante o teste de esforço explicam muito melhor as adaptações sofridas pelo grupo, em função do protocolo de treinamento realizado, do que a FC ao final do teste. Tais resultados devem-se provavelmente a dois aspectos: (1) ao baixo grau de mobilidade dos sujeitos e seu elevado estado de sedentarismo na fase pré-treinamento, o que sem dúvida foi um fator determinante nos resultados muito baixos nessa fase do estudo; (2) a boa adequação entre densidade, volume e intensidade do protocolo de treinamento intervalado, o que o torna eficiente em aumentar o estado de condicionamento de indivíduos com lesão medular. Eficiência essa decorrente também da grande adaptação ao meio aquático demonstrada por todos os sujeitos, devido provavelmente ao uso do colete de flutuação, que propicia maior sensação de segurança no meio líquido, e do aumento da força muscular.

Conforme Zoeller et al. ${ }^{8}$ esclarecem, o ganho de força muscular exerce influência positiva nos exer- 
cícios de potência e resistência aeróbia em indivíduos com lesão medular. Provavelmente, o nosso protocolo, por ser composto por exercícios em domínio moderado a intenso, com significativo componente anaeróbio, pode ter sido determinante de aumento no ganho de força muscular, o que, segundo os autores acima, pode interferir positivamente no condicionamento físico aeróbio, evidenciado em nosso estudo, pelas respostas encontradas no tempo de duração e na distância percorrida.

Ainda de acordo com os autores, o aumento da força muscular reduz a fadiga muscular e o indivíduo consegue realizar uma atividade física por períodos mais longos. Tal fato pôde ser observado pelos resultados de melhora significativa de tempo e duração dos exercícios, sem paralela alteração da percepção subjetiva de esforço. O que nos indica que o protocolo de treinamento intervalado em natação empregado no estudo, com os sujeitos lesados medulares, foi eficiente neste aspecto.

Em outro estudo com sujeitos da população em geral, King ${ }^{48}$ observou que treinamento intervalado com intensidade submáxima mostrou-se mais eficiente do que o treinamento contínuo, de baixa intensidade, com relação à melhora da composição corporal, do condicionamento físico e da taxa metabólica de repouso.

Isso evidenciou que o protocolo intervalado, com intensidade mais elevada, se praticado regularmente, promove um aumento contínuo no gasto de energia maior que o treinamento contínuo de baixa intensidade, pelo menos no estágio inicial do treino, visto que os sujeitos eram sedentários ou realizam muito pouco exercício cardiovascular antes do treinamento ${ }^{48}$, assim como no nosso estudo.

Resultados e considerações semelhantes são apresentados por Navarro ${ }^{49}$ em indivíduos da população em geral. Neste caso, o autor afirma que o treinamento intervalado pode aumentar as possibilidades funcionais do coração, as quais constituem o principal fator limitador do nível de produtividade aeróbica. Além de propiciar ao nadador oportunidade de executar o nado com intensidades mais altas antes do aparecimento da acumulação do ácido láctico e ainda que, quanto maior for a intensidade do trabalho, melhor também será o aperfeiçoamento da capacidade anaeróbica.

O protocolo intervalado com uso de colete de flutuação também mostrou ser adequado para programas de natação realizados em grupo e, em especial, de indivíduos com imobilidade de membros inferiores, pois o grupo respondeu de forma homogênea ao aumento gradativo da intensidade, não havendo necessidade de prescrição individualizada de programa de condicionamento físico.

Como o enfoque no nosso estudo não foi analisar especificamente os parâmetros de VO2máx. e de intensidade, a falta de um teste específico para avaliar o condicionamento físico na água não prejudicou a interpretação geral dos resultados. A utilização da Escala de Borg foi suficiente para que atingíssemos nosso objetivo de verificar a influência do referido protocolo de treinamento, sobre a melhora o estado de condicionamento físico e as alterações de algumas variáveis bioquímicas.

Como o nível e o tipo de lesão não foram fatores de exclusão no GT, mas sim a mobilidade suficiente para executar a braçada do nado peito e costas, a amostra se mostrou bastante diversificada, incluindo sujeitos com nível de lesão de L2 a C4 (Tabela1), embora nenhum dos sujeitos apresentasse mobilidade de membros inferiores.

Nesse sentido, é importante se considerar a heterogeneidade do grupo estudado, no que diz respeito ao tempo e o nível de lesão, além da idade. Fato este observado em vários estudos relacionados a essa população $0^{6,9,12,13,20,26}$.

A amostra disponível para o estudo foi reduzida, por se tratar de uma população isolada socialmente que tem acesso restrito tanto a prática de atividades esportivas, quanto aos locais públicos. Esses aspectos dificultaram o contato com os possíveis voluntários, além do que, por se tratar de pessoas que normalmente apresentam complicações de saúde decorrentes da lesão medular, muitos não se disponibilizaram a participar de um estudo experimental de várias semanas.

\section{Conclusão}

Os resultados do presente estudo sugerem implicações práticas para a promoção de saúde de indivíduos com lesão medular, uma vez que, o protocolo intervalado de natação, com intensidade de moderada a intensa, proporcionou alterações bioquímicas desejáveis nos valores de HDL-colesterol e melhora significativa do estado de condicionamento físico dos sujeitos estudados.

Apesar de considerar as limitações do presente estudo, o protocolo adaptado às condições físicas apresentadas pelos sujeitos mostrou-se adequado a indivíduos com lesão medular iniciantes em natação. 
Dessa forma, o emprego de novos protocolos de treinamento aquático, com duração mais longa e com controle mais aprimorado da intensidade do trabalho por meios específicos, são sugestões importantes para o desenvolvimento de estudos futuros envolvendo pessoas com lesão medular podendo revelarnos respostas mais esclarecedoras.
São necessárias mais pesquisas para verificar os efeitos do condicionamento físico de indivíduos com lesão medular e, consequentemente, para análises mais precisas das mudanças positivas na saúde resultantes de programas de exercícios específicos para essa população.

\begin{abstract}
Introduction: The lack of physical exercises generated by immobilization of the lower limbs leads to changes in body composition that are generally associated with the imbalance of metabolic rate coupled with a sedentary status, which can result in obesity, diabetes mellitus and cardiovascular disease. Therefore, the improvement of physical fitness can contribute to promoting health and quality of life for these patients. As there is a very small number of research in this direction, our purpose was to investigate the effects of an adapted swimming program in protocol interval, for people with spinal cord injury, aiming to verify the improvement of your fitness and, consequently, some biochemical variables important for health. Methodology: The study included 17 subjects with spinal cord injury, sedentary, divided into two groups: 11 participants in the training group (TG) and 6 in control group (CG). TG was applied by a protocol of interval training in swimming for eight consecutive weeks, three times a week. The protocol employed a stroke of breaststroke in work periods of moderate to severe, and stroke in the backstroke, in periods of active recovery. The CG has not participated in any physical activity. Both groups were collecting blood for biochemical analysis, before (evaluation) and after (revaluation) the swimming program. Results and Discussion: The concentrations of triglycerides, total cholesterol and LDL-cholesterol showed no significant changes in assessment for reassessment in both groups. However the TG, the level of HDL-cholesterol were significant differences $(p=0,0110)$, showing an improvement in posttraining, which did not occur in the CG. With respect to the state of fitness, the results revealed a significant difference in relation to time and distance covered in water when compared with the pre-training $(p<0,001)$, showing a great improvement in the ability to shift with the stroke of breaststroke and a significant improvement in cardiorespiratory function. Conclusion: The swimming program interval used, with moderate to severe intensity, can even in a short period of time, promote positive changes in HDLcholesterol in individuals with spinal cord injury studied, and substantially improve your fitness.
\end{abstract}

Keywords: Spinal Cord /Injuries. Swimming. Interval training. Physical Fitness. Cholesterol, HDL. Health Promotion.

\section{Referências bibliográficas}

1. Haddad S, Silva PRS, Barretto ACP, Ferraretto I. Efeito do Treinamento Físico de Membros Superiores Aeróbio de Curta Duração no Deficiente Físico com Hipertensão Leve. Arq Bras Cardiol. 1997; 69: 169-73.

2. Lavis TD, Scelza WM. Cardiovascular health and fitness in person with spinal cord injury. Phys Med Rehabil Clin North Am. 2007; 18:317-31.

3. Janssen TWJ, van Oers CAJM, van Kamp GJ, TenVoorde BJ, van der Woude LHV, Hollander AP. Coronary heart disease risk indicators, aerobic power, and physical activity in men with spinal cord injuries. Arch Phys Med Rehabil. 1997; 78: 697-705.

4. Dallmeijer AJ, van der Woude LHV, van Kamp GJ, Hollander AP. Changes in lipid, lipoprotein and apolipoprotein profiles in persons with spinal cord injuries during the first 2 years postinjury. Spinal Cord. 1999; 37: 96-102.

5. Jacobs PL, Nash MS. Exercise recommendations for individuals with spinal cord injury. Sports Med.. 2004; 34: 727-51.
6. Jones LM, Legge M, Goulding A. Factor analysis of the metabolic syndrome in spinal cord-injured men. Metabolism 2004; 53:1372-7. doi:10.1016/j.metabol.2004.04.013

7. Garshick $E$ et al. A prospective assessment of mortality in chronic spinal cord injury. Spinal Cord. 2005; 43:408-16.

8. Zoeller RF, Riechman SE, Dabayebeh IM, Goss FL, Robertson RJ, Jacobs PL. Relation between muscular strength and cardiorespiratory fitness in people with thoracic-level paraplegia. Arch Phys Med Rehabil. 2005; 86: 1441-6. doi:10.1016/ j.apmr.2004.11.032

9. Kocina P. Body composition of spinal cord injured adults. Sports Med., Auckland 1997; 23:48-60.

10. Bauman WA, Spungen AM, Raza M, Rothstein J, Zhang RL, Zhong YG, et al. Coronary artery disease: metabolic risk factors and latent disease in individuals with paraplegia. Mt Sinai J Med. 1992; 59:163-8.

11. I Diretriz Brasileira de Diagnóstico e Tratamento da Síndrome Metabólica. Arq Bras Cardiol., São Paulo 2005; 84(suppl 1): $1-28$. 
12. Durán FS, Lugo L, Ramirez L, Eusse E. Effects of an Exercise Program on the Rehabilitation of Patients With Spinal Cord Injury. Arch Phys Med Rehabil. 2001; 82: 1349-54. doi:10.1053/apmr.2001.26066

13. Silva RC, Tirapegui J, Ribeiro SML, Pires ISO. Estudo Controlado da Influência da Atividade Física em Fatores de Risco para Doenças Crônicas em Indivíduos Lesados Medulares paraplégicos do sexo masculino. Rev Bras Educ Fís Esp.. São Paulo 2004; 18: 169-77.

14. Quintana R, Neiva CM. Fatores de Risco para Síndrome Metabólica em Cadeirantes - Jogadores de Basquetebol e Não Praticantes. Rev Bras Med Esporte. 2008; 14:188-91.

15. Paeslack V. Esporte para os Paraplégicos. In: Hullemann, K.D. (Org.). Medicina Esportiva: Clínica e Prática. São Paulo: EPU/Educação Física da Universidade de São Paulo, 1978, cap.19, p. 292-9.

16. Meier M. Atividade Física para Deficiente. Ministério da Educação e Cultura. Secretaria de Educação Física e Desportos. Brasília: SEED/MEC, 1981.

17. YMCA of the USA. Aquatics for Special Populations. Champaign: Human Kinetics Publishers Inc. 1987.

18. Adams RC, Daniel A, Mc Cubbin JA, Rullman L. Jogos, Esportes e Exercícios para o Deficiente Físico. 3aㅡ ed. São Paulo: Manole, 1985.

19. Mauerberg-deCastro E. Atividade física adaptada. São Paulo: Tecmedd, 2005. 555p.

20. Silva MCR, Oliveira RJ, Conceição MIG. Efeitos da natação sobre a independência funcional de pacientes com lesão medular. Rev Bras Med Esporte. 2005; 11: 251-6.

21. Gorgatti MG, Teixeira L. Deficiência Motora. In: Teixeira L. Atividade Física Adaptada e Saúde - da teoria à prática. São Paulo: Phorte, 2008. cap.17, p.377-99.

22. Chatard JC, Lavoie JM, Ottoz H, Randaxhe P, Cazorla G, Lacour JR. Physiological aspects of swimming performance for persons with disabilities. Med Sci Sports Exerc. 1992; 24: $1276-82$.

23. Janssen TWJ, Dallmeijer AJ, Veeger D, Woude LHV. Normative values and determinants of physical capacity in individuals with spinal cord injury. J Rehabil Res Dev. 2002; 39: 29-39.

24. Bizzarini E, Saccavini M, Lipanje F, Magrin P, Malisan C, Zampa A. Exercise prescription in subjects with spinal cord injuries. Arch Phys Med Rehabil. 2005; 86: 1170-5. doi:10.1016/ j.apmr.2004.11.014

25. Capoor J, Stein AB. Aging with spinal cord injury. Phys Med Rehabil Clin North Am. 2005; 16:129-61. doi:10.1016/ j.pmr.2004.06.016

26. Haisma JA, van der Woude LHV, Stam HJ, Bergen MP, Sluis TAR, Bussmann JBJ. Physical capacity in wheelchair-dependent persons with a spinal cord injury: a critical review of the literature. Spinal Cord. 2006; 44: 642-52.

27. Barros Filho T.E.P., Oliveira R.P., Kalil E.M., Prada F.S. Associação Americana de Lesões Medulares - Normas para a classificação neurológica e funcional das lesões da medula espinhal. Rev Bras Ortop. 1994; 29: 99-106.

28. Moura RAA. Colheita de material para exames de laboratório: assegurando a qualidade dos serviços no laboratório clínico. 1. ed. São Paulo: Atheneu, 1998. cap. 4 e 26, p. 29-65 e 193-218.

29. Motta VT. Bioquímica clínica para o laboratório: princípios e interpretações. 4. ed. São Paulo: Robe, EDUCS - Caxias do Sul, 2003. cap. 5, p.43-50; cap. 7, p.59-74; cap. 10, p.135-58.

30. Friedewald WT, Levy RI, Fredrickson DS. Estimation of the Concentration of Low-Density Lipoprotein Cholesterol in
Plasma, Without Use of the Preparative Ultracentrifuge. Chemistry. 1972; 18: 499-502.

31. Porto M, Gostardo D, Santos D, Neiva CM. Aplicação de Escala de Percepção Subjetiva de Esforço no Treinamento com Pesos. Saude Rev. - UNIMEP 2008; 23:37-43.

32. Graef FI, Kruel LF. M. Frequência cardíaca e percepção subjetiva do esforço no meio aquático: diferenças em relação ao meio terrestre e aplicações na prescrição do exercício uma revisão. Rev Bras Med Esporte. 2006; 12: 221-8.

33. Mendonça GV, Pereira FD. Controle de níveis de intensidade de esforço pela Escala de Borg em atletas iniciados na modalidade de remo indoor. Rev Bras Educ Fís Esporte Lazer e Dança, 2007; 2:39-47.

34. Triola MF. Introdução à estatística. 9 ed. Rio de Janeiro: LTC, 2005. 410p.

35. Varady KA, Jones PJH. Combination diet and exercise interventions for the treatment of dyslipidemia: an effective preliminary strategy to lower cholesterol levels? J Nutr. 2005; 135: $1829-35$

36. Leon AS, Sanchez OA. Response of blood lipids to exercise training alone or combined with dietary intervention. Med Sci Sports Exerc. 2001; 33(suppl 6): 502-15.

37. Oliveira DM, Santos D, Silva LAGL, Neiva CM. Modulação de perfil lipídico e glicemia de ratos diabéticos. Investigação Revista Científica da Universidade de Franca. 2007; 7: 5360.

38. Yuan G, Al-Shali KZ, Hegele RA. Hypertriglyceridemia: its etiology, effects and treatment. CMAJ 2007; 176: 1113-20.

39. Kraus WE et al. Effects of the amount and intensity of exercise on plasma lipoproteins. N Engl J Med. 2002; 347:1483-92.

40. Washburn RA, Figoni SF. High density lipoprotein cholesterol in individuals with spinal cord injury: The potential role of physical activity. Spinal Cord. 1999; 37: 685-95.

41. Rennie KL, McCarthy N, Yazdgerdi S, Marmot M, Brunner E. Association of the metabolic syndrome with both vigorous and moderate physical activity. Int J Epidemiol. 2003; 32: 600-6.

42. Schwingel $A$ et al. Lower HDL-cholesterol among healthy middle-aged Japanese-Brazilians in São Paulo compared to Natives and Japanese-Brazilians in Japan. Eur J Epidemiol. 2007; 22:33-42.

43. Pádua S, Neiva CM, Tonello MGM, Araújo ECF. Treinamento físico como método terapêutico e controle clínico do diabetes. Lecturas Educación Física y Deportes 2007; 12: 1/1.

44. Kimur T, Kotajima N, Kanda T, Kuwabara A, Fukumura $\mathrm{Y}$, Kobayashi I. Correlation of circulating interleukin-10 with thyroid hormone in acute myocardial infarction. Res Commun Mol Pathol Pharmacol 2001; 110: 53-8.

45. Harvey CB, Williams GR. Mechanism of thyroid hormone action. Thyroid 2002; 12: 441-6.

46. Pereira RJ et al. Contribuição dos domínios físico, social, psicológico e ambiental para a qualidade de vida global de idosos. Rev Psiquiatr RS 2006; 28: 27-38.

47. Bodner ME, Rhodes EC. A review of the concept of the heart rate deflection point. Sports Med. 2000; 30: 31-46.

48. King JW. A comparison of the effects of interval training vs. continuous training on weight loss and body composition in obese pre-menopausal women. A thesis presented to the faculty of the Department of Physical Education, Exercise, and Sports Science East Tennessee State University. May, 2001.

49. Navarro FV. La Resistencia. Madrid, Ed. Gymnos, 1998. 